\title{
Haguenau nid d'espions : la correspondance chiffrée d'un agent secret (novembre-décembre 1516)
}

\section{Georges Bischoff}

\section{(2) OpenEdition}

Journals

Édition électronique

URL : http://journals.openedition.org/rbnu/1492

DOI : 10.4000/rbnu. 1492

ISSN : 2679-6104

Éditeur

Bibliothèque nationale et universitaire de Strasbourg

\section{Édition imprimée}

Date de publication : 1 mai 2016

Pagination : 36-45

ISBN : 9782859230623

ISSN : 2109-2761

\section{Référence électronique}

Georges Bischoff, «Haguenau nid d'espions : la correspondance chiffrée d'un agent secret (novembredécembre 1516) », La Revue de la BNU [En ligne], 13 | 2016, mis en ligne le 01 mars 2020, consulté le 16 décembre 2020. URL : http://journals.openedition.org/rbnu/1492 ; DOI : https://doi.org/10.4000/ rbnu. 1492 


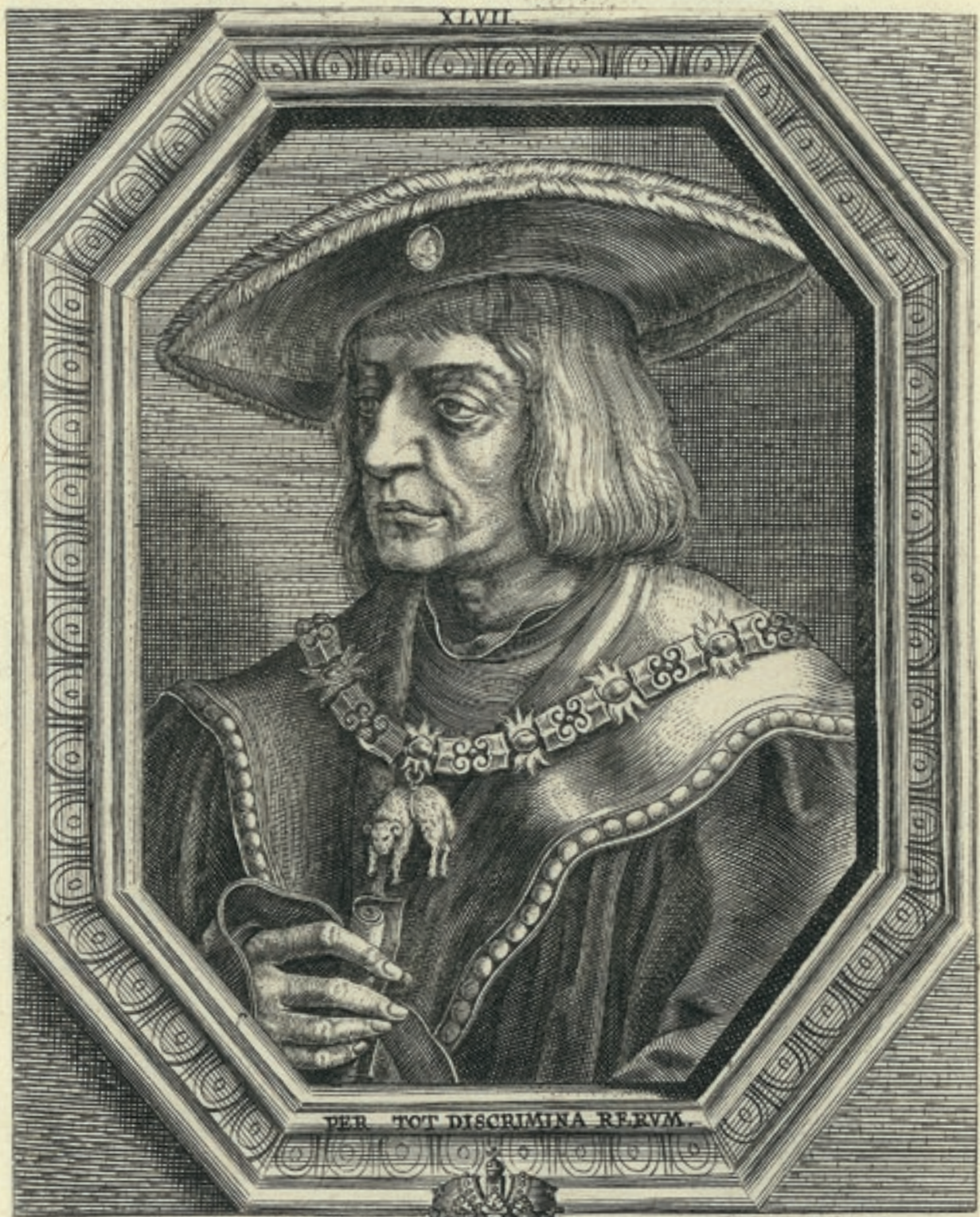

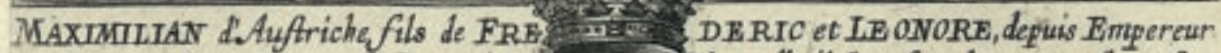
1. du nom, B.fourfa MARIB de Bourgoigne, remarier. Il trefpaffa en Ianuier lan enterrie aupres de fa Mere, laiffant

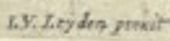

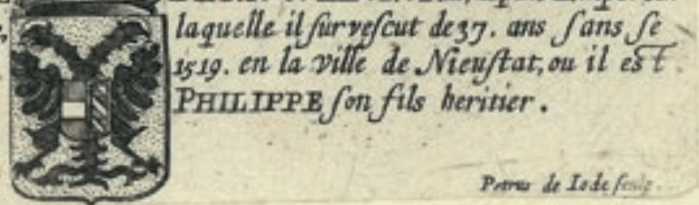




\section{HAGUENAU NID D'ESPIONS : la correspondance chiffrée d'un agent secret (novembre-décembre I5I6)}

$\mathrm{U}$

n épisode majeur de l'histoire de l'Europe s'est joué à Haguenau en novembre et en décembre 1516, il y a tout juste cinq cents ans. L'année précédente, François I ${ }^{\text {er }}$ avait vaincu les Suisses à Marignan (13-14 septembre 1515), en balayant la Sainte Ligue montée par le pape avec l'appui de l'Angleterre, de l'Espagne et de l'Empire. Le jeune roi était désormais le maître de Milan et, aux côtés de Venise, l'arbitre de l'Italie toute entière. Les Confédérés avaient repassé les Alpes pendant que le souverain pontife Léon $\mathrm{X}$ acceptait la tutelle de l'Église de France par le pouvoir royal lors du concordat de Bologne, le 16 août 1516 .

Ce retournement était le prélude d'une recomposition d'autant plus profonde que les coalisés de la veille n'avaient subi que des déconvenues. La mort de Ferdinand le Catholique (23 janvier 1516) donnait l'Espagne à son petit-fils Charles de Bourgogne, alors âgé de quinze ans, mais celui-ci n'était pas le bienvenu en Castille parce qu'il avait été élevé aux Pays-Bas et qu'il était l'héritier de la Maison d'Autriche dont le chef était son autre grandpère, l'empereur Maximilien ${ }^{\text {er }}$. Ce dernier avait bien tenté de reprendre l'offensive en Lombardie, en partant du Tyrol, mais il avait dû s'arrêter à quelques lieues de Milan, saisi par la panique devant la défection de ses propres soldats qu'il n'avait plus les moyens de payer et, dit-on, à la suite d'un cauchemar au cours duquel il avait vécu à la fois la mort de son bisaïeul Léopold III face aux Confédérés suisses, à la bataille de Sempach (1386) et celle de son beau-père Charles le Téméraire devant Nancy, en présence des mêmes ennemis (1477). Quant à Henry VIII d'Angleterre, dont la promenade militaire en France en 1513 n'était plus qu'un vieux souvenir, il n'était plus sur le théâtre des opérations, mais on comptait sur lui pour financer l'effort de guerre de l'empereur. En mai 1516, c'est dans cette perspective que ce dernier avait tenté d'ouvrir un deuxième front en attaquant le duché de Lorraine dont le duc avait été l'un des vainqueurs de Marignan : confiée au comte Gangolphe de Geroldseck et au capitaine mercenaire Franz von Sickingen, sous couvert d'une guerre privée, cette campagne s'était traduite par l'invasion des possessions lorraines du versant alsacien des Vosges (Saint-Hippolyte et le Val de Lièpvre), par une incursion dans la vallée de la Sarre et un coup de main sur le petite ville de Conflans en Bassigny, sur la route de la Champagne, de façon à permettre la jonction des troupes venues du nord et de l'est en bloquant l'arrivée d'éventuels renforts français.

La première phase de ce Kriegsspiel s'était passée comme prévue mais, comme les soldes promises par Henry VIII n'arrivaient pas, les milliers de lansquenets de Geroldseck et Sickingen avaient fondu comme neige au soleil, et il avait fallu quelques jours à peine pour que le duc Antoine de Lorraine rétablisse la situation avec l'aide de ses alliés français, pratiquement sans coup férir. En juillet 1516 les Vosges s'étaient retrouvées frontière, comme si l'Alsace était, dorénavant, le dernier bastion de l'Empire du côté de l'ouest - ce qu'ânonnaient déjà les humanistes de la génération de Wimpheling.

Au cours de l'été 1516, cependant, les pulsions belliqueuses des uns et des autres évoluent vers l'apaisement. Les Suisses, définitivement hors course, s'entendent avec François I ${ }^{\text {er }}$ pour régler leurs derniers contentieux : l'or français a des vertus bénéfiques. Le pape a fait la paix et, de son côté, l'archiduc Charles de Bourgogne, devenu Charles I ${ }^{\text {er }}$ d'Espagne, joue la carte de l'amitié avec la France pour avoir les mains libres aux Pays-Bas et au sud des Pyrénées : conseillé par sa tante, Marguerite 
d'Autriche, duchesse douairière de Savoie, il était resté à l'écart des hostilités ouvertes en 1512. Résultat ? Le traité de Noyon du 13 août 1516, par lequel le roi de France et celui qui sera bientôt son rival s'entendent sur leurs prétentions respectives sur le royaume de Naples et celui de Navarre, et rêvent d'une paix étendue à l'ensemble de l'Europe. Seuls Henry VIII et Maximilien I ${ }^{\text {er }}$ traînent la jambe, le second étant bien décidé à continuer la guerre et, pour ce faire, à mobiliser ses sujets allemands. C'est dans ce climat d'incertitudes que l'Alsace accueille le souverain dans la deuxième quinzaine de novembre et tout au long du mois de décembre, et que la résidence impériale de Haguenau devient le centre d'un ballet diplomatique sans précédent.

Curieusement, ces événements n'ont pas laissé beaucoup de traces dans la région : leur dimension se situe à une échelle différente et les documents susceptibles de les saisir se trouvent dans des archives lointaines. Les rares historiens qui les ont vus, à Paris, Rome, Venise, Londres, Vienne ou ailleurs n'ont pas eu l'occasion de les confronter; ils n'en ont publié que de courts extraits, dans les State Papers d'Henry VIII, ou les Diarii di Marino Sanuto vénitiens, des collections diplomatiques que possède, heureusement, la BNU. L'un des meilleurs observatoires du premier quart du $16^{\mathrm{e}}$ siècle est le fonds de la Chambre des comptes de Lille, aux Archives départementales du Nord : c'est là qu'a été conservée la correspondance de Marguerite d'Autriche (1480-1530), fille de Marie de Bourgogne et de Maximilien $\mathrm{I}^{\mathrm{er}}$ et régente des Pays-Bas " bourguignons " (et de la Franche-Comté) entre 1507 et 1515, date de l'accession au pouvoir de son neveu Charles, puis de 1519 à sa mort. Les dizaines de milliers de lettres qu'elle a reçues à la cour de Malines sont une véritable mine d'or pour l'historien, mais seules quelques centaines d'entre elles ont réellement été utilisées, par des savants comme Godefroy ${ }^{1}$ et Le Glay ${ }^{2}$. Des recherches récentes nous ont donné l'occasion de consulter ces pièces inédites, et nous y avons notamment découvert un certain nombre de dépêches d'un des agents de la duchesse à la cour de Maximilien, le secrétaire Loys Maroton, dans lequel on peut voir un diplomate officieux aussi bien qu'un espion.

Première remarque : il ne se passe pas deux jours sans que ce personnage donne des nouvelles à Marguerite d'Autriche, en clair ou en langage codé. Il peut s'agir d'un court feuillet ou d'un rapport plus substantiel, qui emprunte un système permanent de messagerie - la poste, dont on peut rappeler qu'elle a été organisée par l'Italien François de Taxis (1450-1517) en 1489, avec des relais tous les $28 \mathrm{~km}$, un "hub", une plaque tournante, près de Francfort et son terminal à Bruxelles - ou des courriers occasionnels. Le système marche dans les deux sens, mais les instructions de la duchesse ne sont pas conservées - sont-elles détruites, pour ne pas tomber dans des mains indiscrètes ? On peut cependant les déduire des réponses qu'elles occasionnent.

\section{Comment déchiffrer un document crypté}

En règle générale, les lettres de Loys Maroton s'ouvrent sur les formules de politesse convenues : elles ne sont jamais intégralement chiffrées. Elles se terminent sur l'indication du lieu et de la date. Le langage secret est employé pour les passages les plus délicats, car l'auteur se sait entouré d'ennemis. Le 2 décembre, il confie : «le pays où je suis n'est pas le pays de la justice et de la liberté ", signifiant par là que le soupçon règne autour de lui et qu'on essaye de le discréditer. Quelque temps plus tôt, il avait été envoyé en mission à Rome et savait, mieux que quiconque, ce qui se tramait entre la France et l'Italie. L'atmosphère de fin de règne dans laquelle baignait la cour impériale était connue de tous : d'un côté, les fidèles allemands du vieil empereur - un certain nombre d'Alsaciens, comme son " ministre des finances" Jacques Villinger, qui rêvaient d'une succession en faveur de Charles d'Espagne, de l'autre, le chassé-croisé des intérêts particuliers, ceux du pape, défendu par l'évêque de Sion, le cardinal Mathieu Schiner qui avait été l'âme de la Sainte Ligue, les amis du roi d'Angleterre, le parti favorable à un accord avec la France, avec le favori du jeune Charles, Guillaume de Chièvres. La voie illustrée par Maroton est celle de Marguerite de Savoie, plus raisonnable que son père Maximilien mais, pour lors, privée de la régence qu'elle exerçait naguère dans l'esprit de ses ancêtres bourguignons. Faut-il l'interpréter comme une volonté d'apaisement général et, corrélativement, comme un prélude à la restauration de la princesse ? Ce qui est sûr, c'est que cette femme entend rester la maîtresse du jeu, et que son influence est déterminante.

C'est pendant le séjour de Loys Maroton à Haguenau que Maximilien et Henry VIII se résignent à sceller la paix avec le roi de France. L'après-guerre de Marignan a été gagné en Alsace.

Avant d'analyser un exemple de ces missives, on donnera quelques éclaircissements sur le code secret employé. Le principe est très simple. L'émetteur et le récepteur disposent chacun d'une grille donnant l'équiva- 


\begin{tabular}{|c|c|c|c|c|c|c|c|c|c|c|c|}
\hline A & B & $\mathrm{C}$ & $\bar{D}$ & $E$ & $F$ & G & $\mathrm{H}$ & I-J-Y & $\mathrm{K}$ & $\mathrm{L}$ & $\mathrm{M}$ \\
\hline$P$ & 0 & 2 & 72 & 9 & $L$ & $f$ & 4 & $x$ & 6 & $\bar{F}$ & 1 \\
\hline$E$ & & & & B & & F & & $q$ & & & \\
\hline 9 & & & & $E$ & & & & $y$ & & & \\
\hline & & & & & & & & 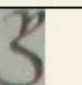 & & & \\
\hline
\end{tabular}

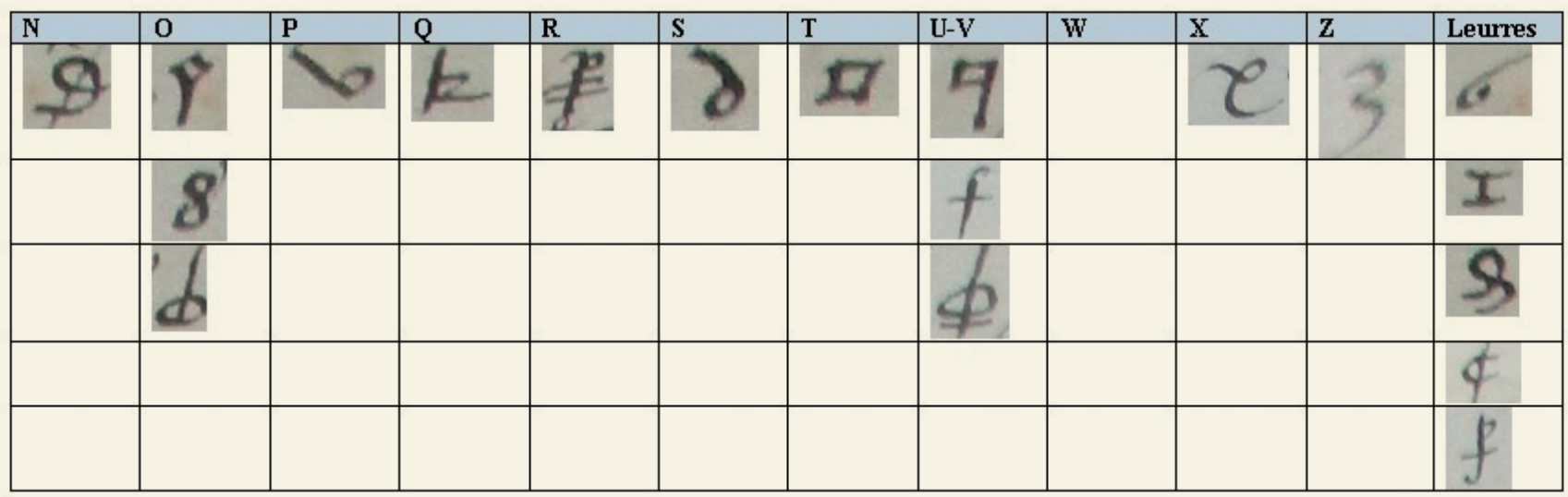

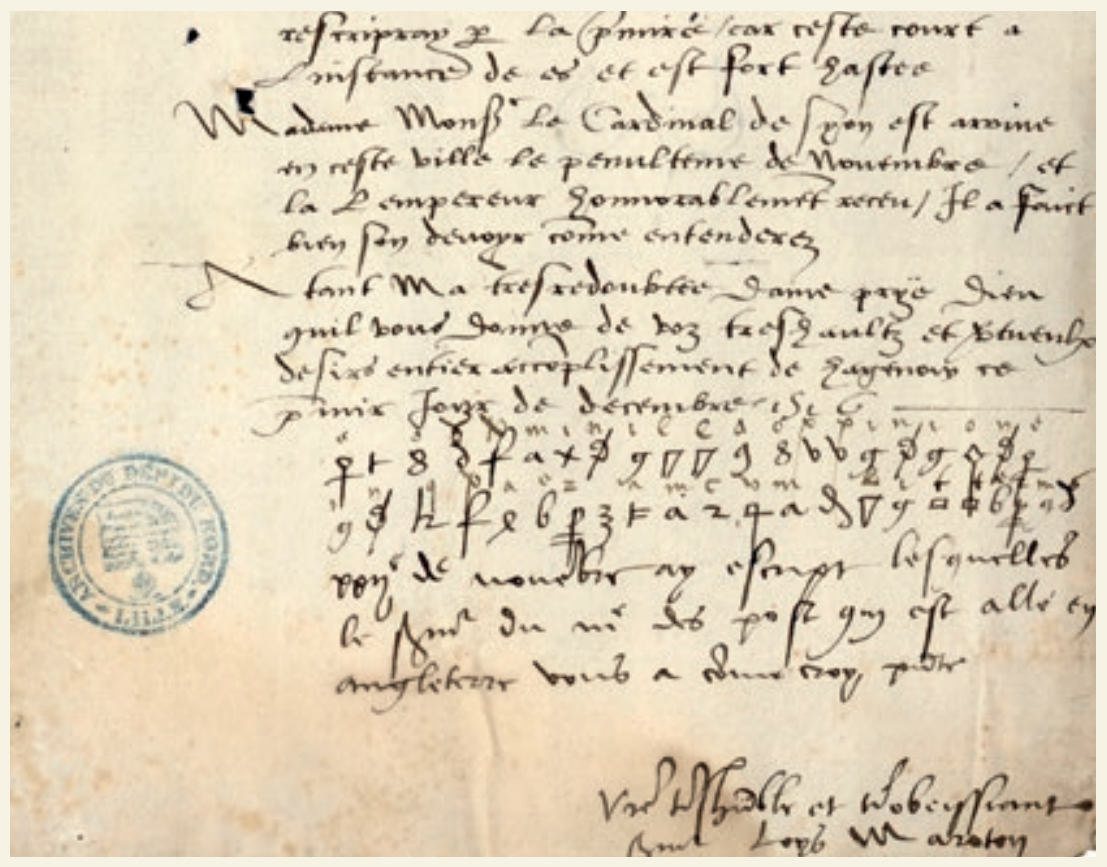

Madame Monsr le cardinal de Syon est arrivé en ceste ville le pénultème de novembre et l'a l'empereur honorablement receu ; il a faict bien son devoyr comme entenderez.

Tant ma très redoublée dame prye Dieu qu'il vous donne de vos très haultz et vertueulx désirs entier accomplissement. De Haguenow ce premier jour de décembre 1516.

Et ${ }^{*}$ sum in illa oppinione in qua eram cum litteris xxii de novembre ay escript lesquelles le serviteur du maître des post qui est allé en Angleterre vous a comme croy porté.

Votre très humble et très obéissant serviteur Loys Maroton

Un passage en latin : extrait d'une lettre de Loys Maroton à l'archiduchesse Marguerite le $1^{\text {er }}$ décembre 1516. Les phrases chiffrées sont reproduites en italique, les fausses lettres remplacées par * (coll. Archives départementales du Nord). 
a traguenan 64 occomber

32136

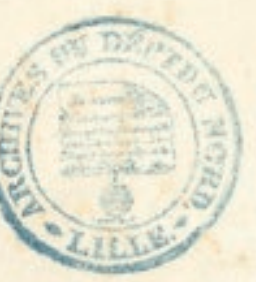

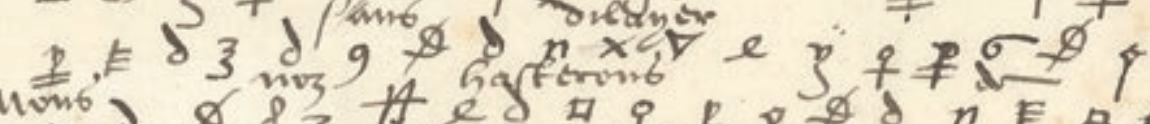

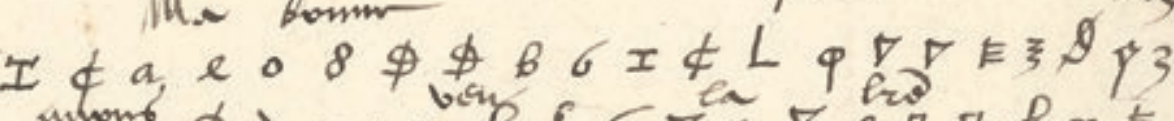
e

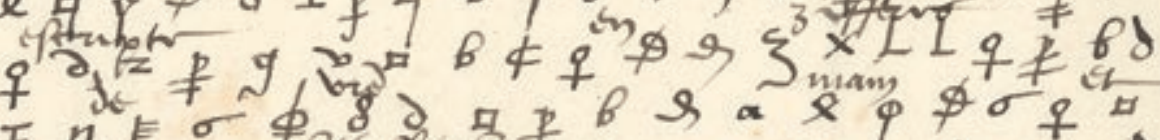

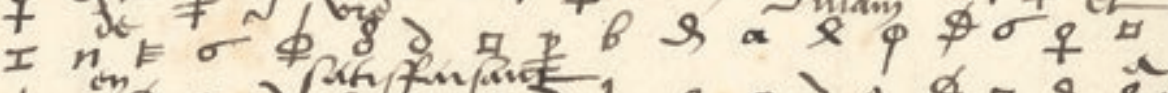

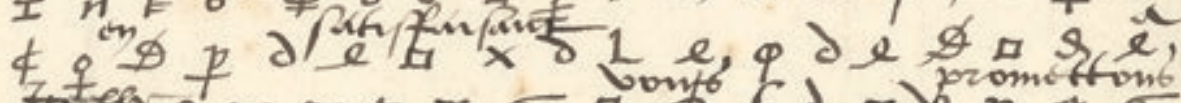

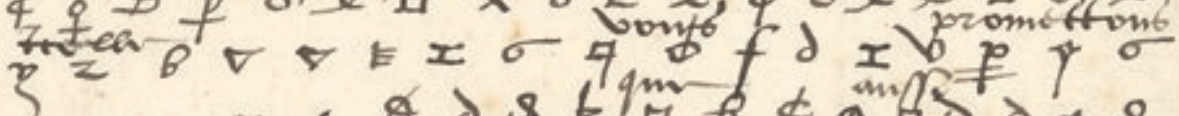

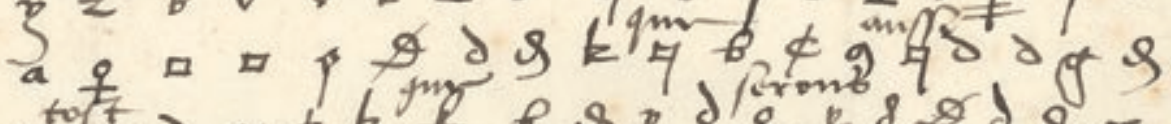

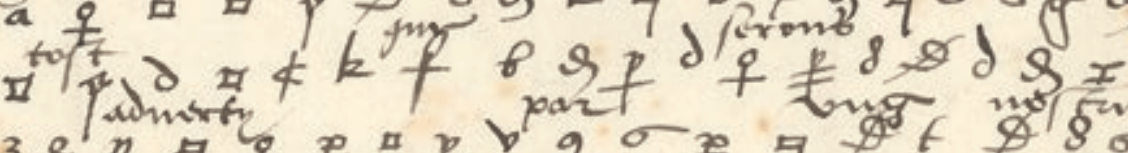

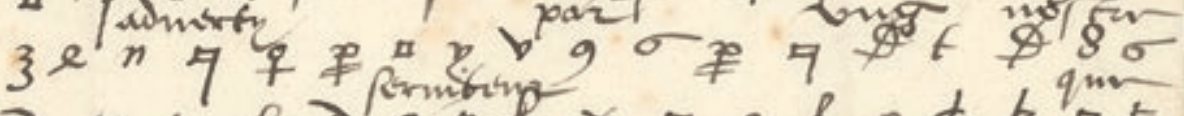

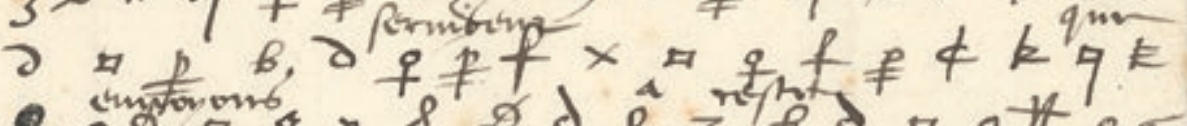

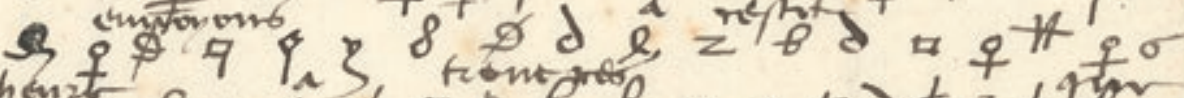

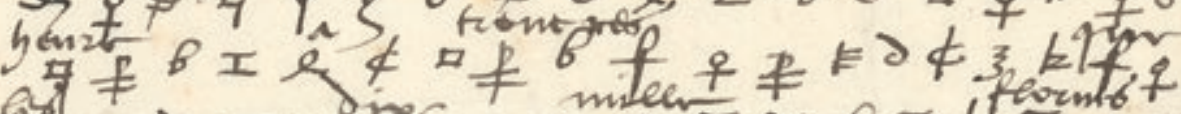

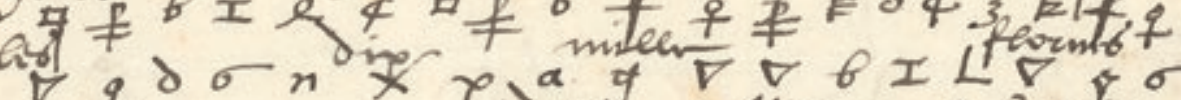

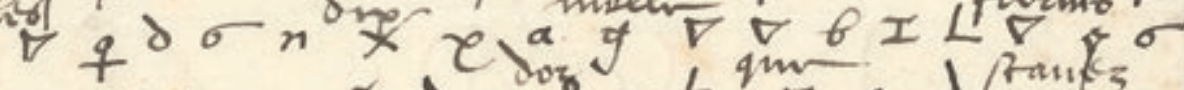

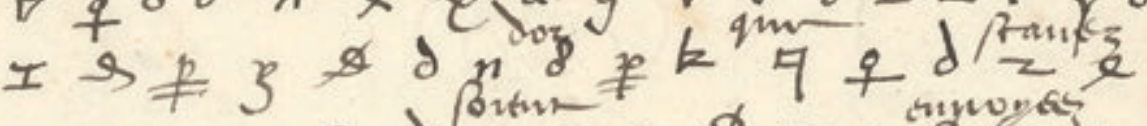

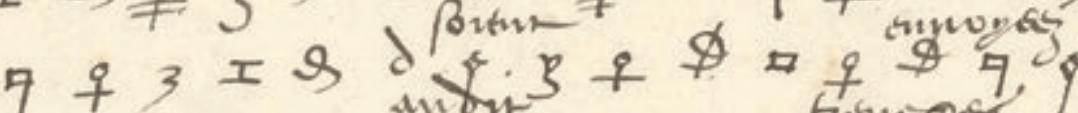
$7+$ andur funcer

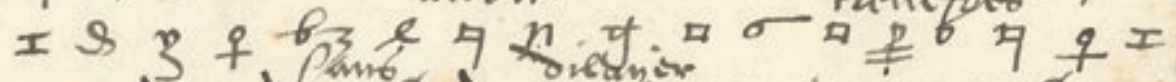

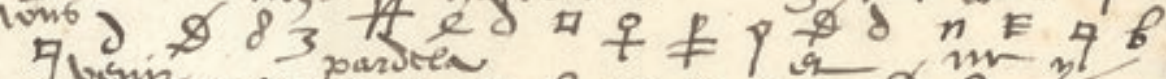

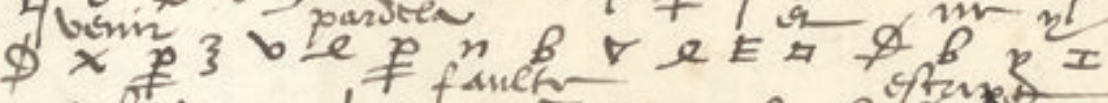

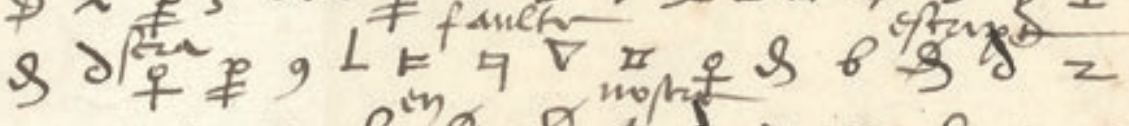

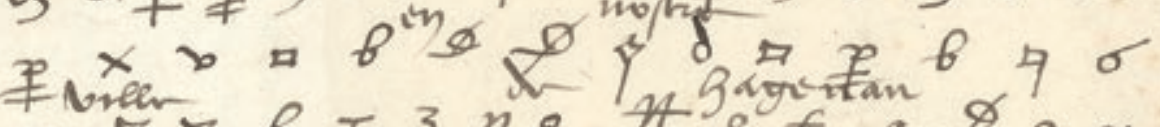

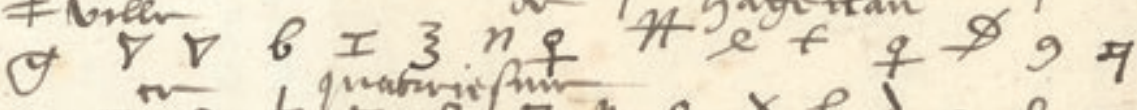

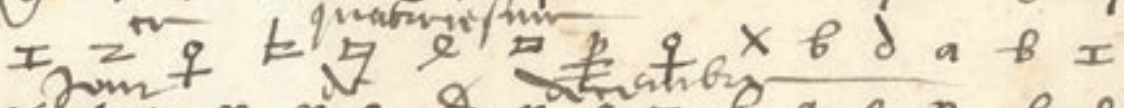

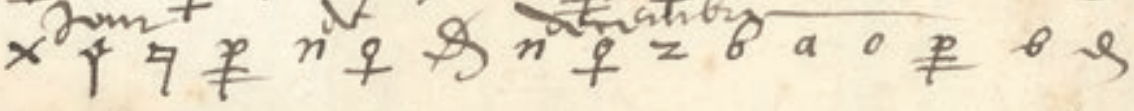

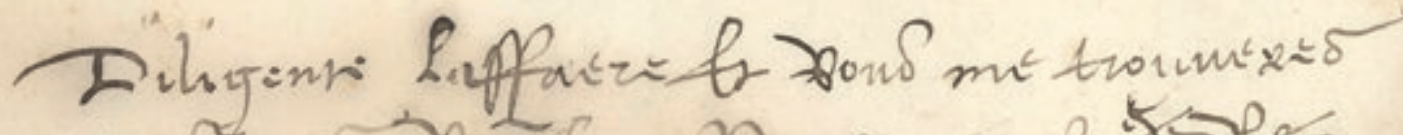

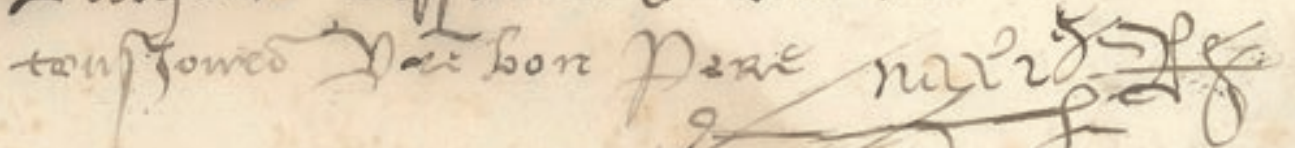
aux Pays-Bas dès qu'il aura reçu les 10 ooo florins (promis par Henry VIII).

Haguenau, 4. 12. 1516 (coll. Archives départementales du Nord). 


\section{TEXTE BRUT}

${ }^{* * *}$ Ma bonne *** fille * noz avons ${ }^{* * * *}$ veu * la levtere escripte ${ }^{* *}$ en zifferes

* de ** vostre * main * et

* en * satisfaisant * a

icelle ${ }^{* *}$ vous ** pro* mettons *** que * aussi * tost * que ** serons ** * adverty pa*r ung nos * tre * serviteur * que * envoyeons a ceste he* ure * $a^{*}$ treveres ${ }^{* *}$ que les * dix mille flo*** rins dor que scavez ** soient envo ** yeez sans dilayer ** nous noz hasterons de venir * par dela et ne $y^{* *}$ sera faulte ${ }^{*} e^{*}$ script en nostre $v^{*} i l l e$ ** de Hagenau * ce quatriesme * jour de * decembre *

\section{Diligente l'affaere} et vous me trouveres tousjours votre bon père Maximilien

\section{TEXTE} ADAPTÉ

Ma bonne fille, noz avons veu la levtere escripte en zifferes de vostre main et en satisfaisant a icelle vous promettons que aussitost que serons adverty par ung nostre serviteur que envoyeons a ceste heure a treveres que les dix mille florins d'or que scavez soient envoyeez sans dilayer nous noz hasterons de venir par dela et ne $y$ sera faulte escript en nostre ville de Hagenau, ce quatriesme jour de decembre

\section{Diligente l'affaere} et vous me trouveres tousjours votre bon père Maximilien

\section{TEXTE}

\section{MODERNISÉ}

Ma bonne fille,

Nous avons vu la lettre

écrite en chiffre de votre main et, en réponse à celle-ci, vous promettons que dès que nous serons averti par un de nos serviteurs que nous envoyons à l'heure même à Trèves, que les dix mille florins que vous savez seront envoyés sans délais, nous nous hâterons de venir par-delà, sans faute. Écrit dans notre ville de Haguenau, ce quatrième jour de décembre.

Diligentez l'affaire, et vous me trouverez toujours votre bon père.

\section{Maximilien}


lent alphabétique des signes utilisés. Le plus souvent, on utilise des caractères spéciaux, apparentés à l'écriture sténographique inventée par Tiron, le secrétaire de Cicéron. Ces abréviations "tironiennes " peuvent s'appliquer à des noms propres, des personnes ou des lieux. La langue habituelle est le français, mais on peut également recourir au latin (comme dans le document p. 39), à l'italien, voire au flamand.

Pour compliquer la lecture, on peut noter la même lettre de différentes manières et même farcir le texte de signes dépourvus de signification, en coupant certaines phrases. Il n'y a pas de ponctuation. Enfin, pour éviter les fuites, on peut changer de codes ou adapter ceux-ci en fonction des correspondants. Bien entendu, le courrier peut être intercepté ou subtilisé : dans le premier cas, il parvient à sa destinataire avec un certain retard, ce qui peut lui mettre la puce à l'oreille. En effet, le déchiffrement est une question de rapidité : plus il est difficile, plus on perd un temps précieux, d'autant que la fréquence des messages peut contrarier la vigilance de l'adversaire.

Pour casser un chiffre, il faut pouvoir reconnaître des mots récurrents ou des combinaisons de lettres sur des lignes dépourvues d'espaces et de ponctuation. Les joueurs de scrabble et les lecteurs de Perec savent que le E est plus courant que le $\mathrm{W}$, en français tout au moins. Par conséquent, c'est un signe récurrent, qui peut facilement s'agglutiner à une autre lettre pour former une diphtongue. De même, les lettres redoublées, ll, $\mathrm{mm}, \mathrm{nn}$ ou tt devraient être aisément repérables. En 1516, Maroton utilise au moins quatre E différents, mais ne maquille pas les lettres redoublées qui sont, fatalement, précédées ou suivies par des voyelles. Les caractères spéciaux désignant des personnes ou des lieux sont, en principe, inintelligibles pour le profane mais, là encore, la palette est suffisamment restreinte pour qu'on puisse les identifier. Finalement, le système le plus efficace consiste à brouiller le texte en y introduisant des passages incohérents, ou même à intoxiquer le lecteur avec des informations fallacieuses. Par chance, le service de décryptage de Marguerite d'Autriche retranscrit presque systématiquement sa lecture sur l'original.

\section{Ce que nous apprend cette correspondance secrète : un exemple}

Rapport de Loys Maroton à l'archiduchesse Marguerite, Saverne, 16 novembre 1516 (voir ill. ci-contre)

Transcription

[1] Madame ceulx de la conté de Ferrette ont estez assamblés et aucuns mauvais espris ont tellement troublé ladite assemblée que les estas de ladite conté requierent à l'empereur que son bon plaisir soit de accepter le traicté fait à Noyon, ce que sa Majesté nullement est delibéré de faire mais a connclu de tenir promes au Roy d'Angleterre.

[2] Madame mess ${ }^{\mathrm{r}}$ Lenard Friscobaldi ${ }^{3}$ sert très mal l'empereur car le Focquer ${ }^{4}$ ne veult donner crédit à ces lettres de changes et at esté l'empereur contraint de se obliger audit Focquer de lui rebourser an fin du présent mois les xxxvi ${ }^{\mathrm{m}}$ florins d'or qui sont envoiés à Vérone au cas que ledit de Friscobald ne faise son debvoir vers le factueur du Focquer à Anvers. Et quant aux autres lettres de changes envoié par ledit Freiscobaldi par ordonnance du Roy d'Angleterre de xiiij ${ }^{\mathrm{m}}$ florins d'or dit Focquer que ne délivrera pas l'argent sans estre adverti que son factueur estant a Anvers soit assuré de ladite servir. De ceste matière par le poste ay escript au conte Ticien ${ }^{5}$ et à Hesdin ${ }^{6}$.

[3] Madame pour Dieu diligentez les dix mille florins d'or en essuant ${ }^{7}$ la lettre que l'empereur vous escript le plus tôt que possible vous sera car je vous promis que l'empereur fera ce que monseigneur le cardinal de Syon ${ }^{8}$ a promis au roy d'Angleterre de la part de sa Majesté.

[4] Madame si Mons ${ }^{\text {gr }}$ de Zevenberghen ${ }^{9}$ n'est pas en court faitte lle venir vers vous car l'empereur se veult servir de lui et a bien entendu Sa Ma[jesté] la lettre qu'il m'a envoyée par ung qui at esté son barbier par la présente vous advertirai ${ }^{10}$ plus au long à la vérité de ceste matère.

[5] Madame l'empereur sera demain au soir entre Hagenau et Landau à six lieues ${ }^{11}$ de Spire.

[6] Jorge Hagen Hacheny fils de Casius est parti par les postez de Friburg le xvj de présent et pourtant que la bogget ${ }^{12}$ courrioit aucun ne ay fait mention de son allé mais sa despèche et instruction est plaine de double entendement. 


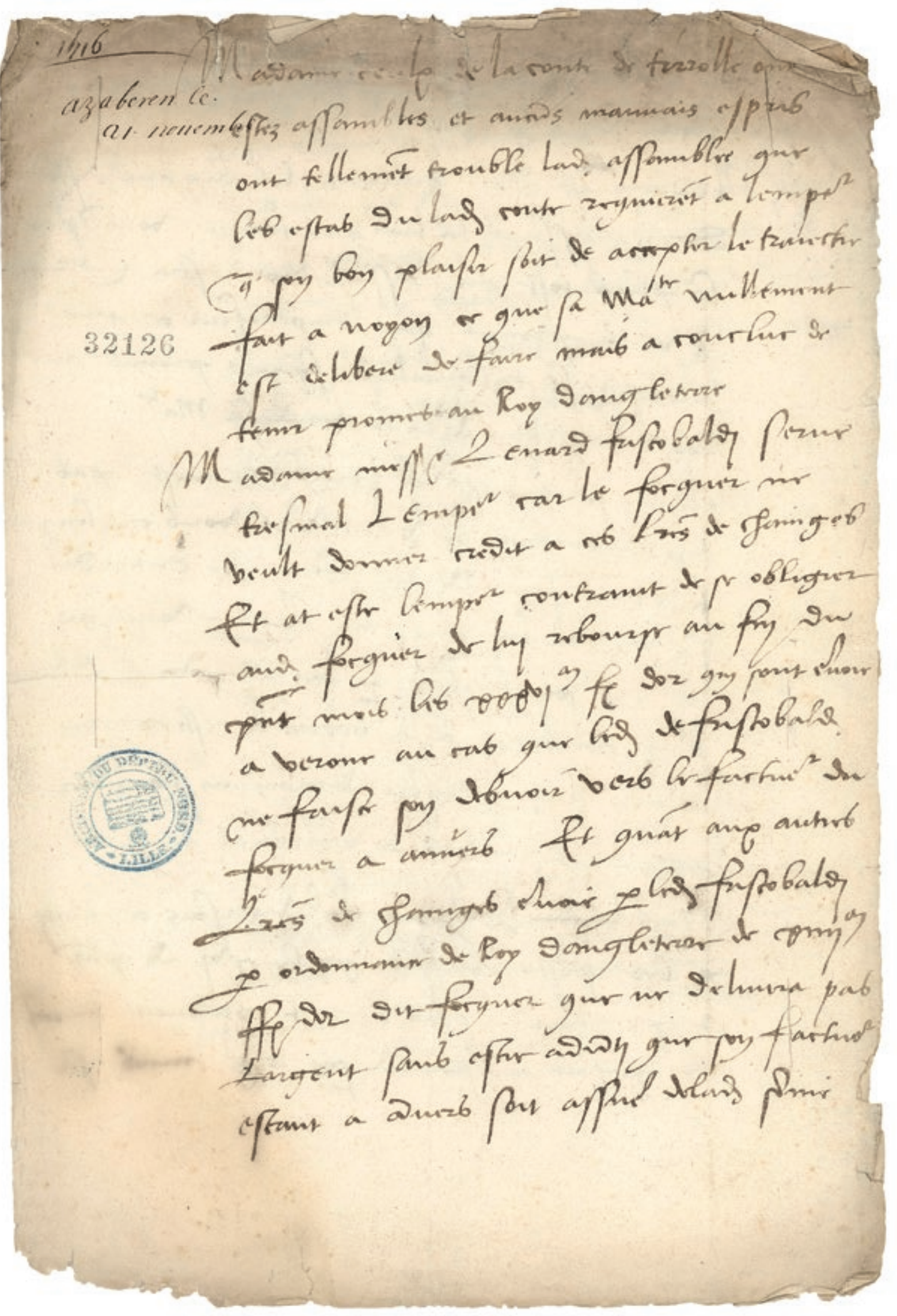

Début du rapport de Loys Maroton (coll. Archives départementales du Nord). 


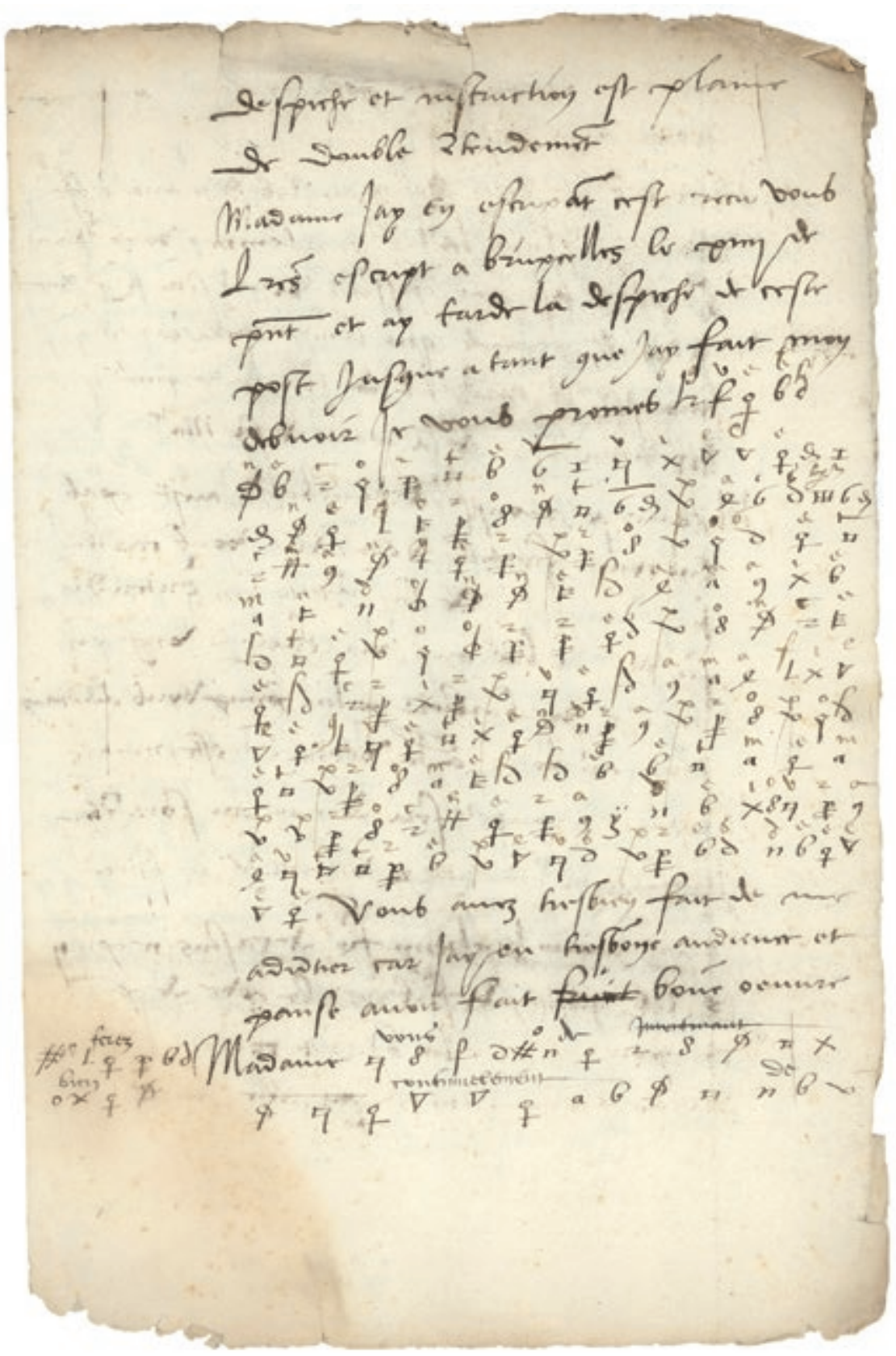

Début du paragraphe 7 , en partie crypté, du rapport de Loys Maroton (coll. Archives départementales du Nord).

[7] Madame j'ay en escrivant cest recu vous lettres escript a Bruxelles le xiiii de présent et ay tardé la despesche de ceste post jusque a tant que j'ay fait mon debvoir et vous promets que Es ${ }^{13}$ ne Corteville ${ }^{14}$ ne feront [..] pa[.] s l'empereur changer propos et m'a donné sa maiesté poor responce escrirpves à ma fille que tiendra propos et promesse et m'en $p$ procheray de iovr a auttre peus près de elle.

[8] Vous avez très bien fait de me adviser car j'ay en très bonne audience et pense avoir fait bonne œuvre.

[9] Madame vous ferez bien de continuellement de depecher postes et escripre lettres de votre main en asseurant
Sa Majesté que sans dangier peult venir par deça et qu’il trouvera le roy catholicque obéissant volontiers d'accomplir ses désirs et que le roy d'Angleterre destendra à son plaisir et ne le laissera point en nécessité d'argent. Quant à moy je tiendrai vous affaires secrètes, vous servant loyalement et seray ennemis de vous ennemis tant que lame me bat au corps sans jamais changier propos.

Escript a Zaberen plus bas que Strasbourg, iii lieues, le xxj de novembre $\mathrm{A}^{\circ} \mathrm{xvj}^{\circ}$ 


\section{Analyse}

À première vue, les nouvelles paraissent un peu décousues puisqu'il est question ici de la mauvaise humeur des sujets de la Maison d'Autriche [1], des embarras d'argent de l'empereur [2], de l'alignement de la politique de celui-ci et du roi d'Angleterre [3], d'une mission à confier à Maximilien de Berghes, seigneur de Zevenbergen [4], des déplacements de l'empereur [5], de l'absence de nouvelles d'un messager parti de Fribourg [6], des instructions reçues de la duchesse et de leur application [7], enfin, des conseils destinés à celle-ci $[8,9]$.

Trois éléments méritent d'être soulignés avec force. En premier lieu, les difficultés financières de l'empereur, qui compte sur l'aide anglaise pour continuer la guerre contre le roi de France. On constate que le banquier florentin d'Henry VIII, Frescobaldi, ne tient pas les engagements de celui-ci et que son correspondant Jacques Fugger d'Augsbourg refuse d'acquitter la lettre de change promise. Le prêt devrait transiter d'Anvers à Augsbourg ou à Strasbourg, par l'intermédiaire de Frédéric Prechter, cité dans une autre lettre. Frescobaldi est au bord de la banqueroute, mais on ne le sait pas encore, et Maroton lui-même n'a pas encore été payé. Dans l'urgence, on insiste sur le règlement rapide de 10 ooo florins.

Le deuxième point concerne les réticences des sujets de la Maison d'Autriche : pour lever de nouveaux impôts, Maximilien a convoqué les trois états des Pays antérieurs, c'est-à-dire le clergé, la noblesse et ce qu'on appelle le "pays " (Landschaft), les représentants des villes et des bailliages de Haute-Alsace, du Sundgau (« le comté de Ferrette ») et de la rive droite du Rhin. Par " mauvais esprits ", il faut comprendre une opposition véhémente, pour ne pas dire un vent de révolte. Au cours des années qui précèdent, le souverain a multiplié ses exigences et ses sujets en ont assez. En Wurtemberg, en 1514, les mêmes causes s'étaient traduites par la mise sous tutelle du duc Ulrich, obligé d'accepter une " grande charte " limitant ses pouvoirs. Ici, on constate que les sujets des Habsbourg font pression pour que Maximilien I ${ }^{\text {er }}$ se rallie, contre son gré, à l'idée d'une paix générale. C'est la preuve, éclatante, de l'existence d'un contre-pouvoir ou, du moins, d'un dialogue, parfois très rude, entre les gouvernants et les gouvernés. La BNU possède le manuscrit du Landschaftbuch ${ }^{15}$ dans lequel sont consignées les décisions prises en commun par ces derniers à partir de 1525 : ce monument de l'histoire régionale attend toujours son historien.

Le dernier point se rapporte aux tractations de l'archiduchesse pour attirer son père aux Pays-Bas.
Apparemment, c'est le principal résultat de la mission de Loys Maroton, à qui il arrive de rencontrer le souverain de la manière la plus discrète. Le 30 décembre 1516, Maximilien quitte Haguenau pour Reichshoffen et Bitche, puis fait route vers le nord, emprunte la Moselle jusqu'à Maastricht et rejoint sa fille à Malines le 20 janvier suivant. Il demeure auprès d'elle et de son petitfils Charles d'Espagne jusqu'à son retour définitif en Allemagne en juin. Dans l'intervalle, tout le monde s'était rallié au traité de Noyon, l'empereur, assagi, et son allié Henry VIII ouvrant la voie à la paix universelle rêvée par Erasme dans sa Querela pacis parue chez Froben, à Bâle, à la fin de l'année $1517 \ldots$

La suite au prochain épisode : Martin Luther à Wittenberg (octobre 1517), la mort de Maximilien (janvier 1519) et l'avènement de Charles Quint à la tête d'un empire où le soleil ne se couche jamais.

\section{Georges Bischoff}

\section{Notes}

1 - Jean Godefroy, Lettres de Louis XII et du cardinal Georges d'Amboise, Bruxelles, 4 vol., 1712

2 - André Joseph Ghislain Le Glay, Correspondance de l'empereur Maximilien $1^{\text {er }}$ et de Marguerite d'Autriche de 1507 à 1519, Paris, 2 vol., 1839, et Négociations diplomatiques entre la France et l'Autriche durant les trente premières années du XVI siècle, 2 vol., Paris, 1845

3 - Banquier chargé par Henry VIII de remettre 60 ooo florins à Maximilien $\mathrm{I}^{\mathrm{er}}$, pour financer sa campagne contre François $\mathrm{I}^{\mathrm{er}}$, Leonardo Frescobaldi (Florence 1485-1529) ne parvint pas à réunir les fonds.

4- Jacob Fugger (1469-1525)

5- Probablement Bartolomeo Tizzone, diplomate piémontais proche de Marguerite d'Autriche

6- Hesdin, probablement Jean d'Ostin dit Hesdin, gouverneur de Béthune, maître d'hôtel de Marguerite de Savoie.

7 - En donnant suite.

8 - Mathieu Schiner (1465-1522), prince-évêque de Sion, principal artisan de l'alliance entre les Confédérés suisses et Jules II et cheville ouvrière de la Sainte Ligue contre la France en 1512-1516.

9 - Maximilien de Berghes, seigneur de Zevenbergen (+ 1545), chevalier de la Toison d'Or en 1516, un proche du futur Charles Quint.

10-Casius [Nicaise] Hackenay est un maître d'hôtel de Charles de Bourgogne, roi d'Espagne.

11- Une lieue d'Allemagne : env. 7,5 km.

12- Bogget (qui a donné budget) : probablement le sac du courrier. Le mot boguet, buggy en anglais, désignant un véhicule rapide à deux roues n'est attesté qu'au premier quart du $19^{\mathrm{e}}$ siècle et dérive vraisemblablement du patronyme du carrossier Boquet.

13- Non identifié : probablement un autre agent diplomatique ou Jean le Sauvage (1455-1518), chancelier de Flandre, seigneur d'Escaubecq, proche de Guillaume de Chièvres.

14- Jean de Courteville, bailli de Lille, diplomate au service de l'archiduc Philippe le Beau puis de Charles de Bourgogne.

15 - MS. 845 